\title{
BMJ Open Exploring human factors in the operating room: a protocol for a scoping review of training offerings for healthcare professionals
}

\author{
Alex Lee (D , ${ }^{1}$ Ben Tipney, ${ }^{2}$ Alexandra Finstad, ${ }^{1}$ Alvi Rahman, ${ }^{3}$ Kirsten Devenny, ${ }^{4}$ \\ Jad Abou Khalil, ${ }^{5}$ Craig Kuziemsky, ${ }^{6}$ Fady Balaa ${ }^{5}$
}

To cite: Lee A, Tipney B, Finstad A, et al. Exploring human factors in the operating room: a protocol for a scoping review of training offerings for healthcare professionals. BMJ Open 2021;11:e044721. doi:10.1136/ bmjopen-2020-044721

- Prepublication history and supplemental material for this paper is available online. To view these files, please visit the journal online (http://dx.doi org/10.1136/bmjopen-2020044721).

Received 12 September 2020 Accepted 26 April 2021

Check for updates

(C) Author(s) (or their employer(s)) 2021. Re-use permitted under CC BY-NC. No commercial re-use. See rights and permissions. Published by BMJ.

For numbered affiliations see end of article.

Correspondence to

Dr Fady Balaa; fbalaa@toh.ca

\section{ABSTRACT}

Introduction Applying human factors principles in surgical care has potential benefits for patient safety and care delivery. Although different theoretical frameworks of human factors exist, how providers are being trained in human factors and how human factors are being understood in vivo in the operating room (OR) remain unknown. The aim of this scoping review is to evaluate the application of human factors for the $\mathrm{OR}$ environment as described by education and training offerings for healthcare professionals.

Methods and analysis This scoping review will follow the Preferred Reporting Items for Systematic reviews and Meta-Analyses extension for Scoping Reviews guidelines. MEDLINE, Embase, PsycINF0, CINAHL, Health and Psychosocial Instruments and ERIC databases were searched on August 2020 from inception to identify relevant studies that describe the content, application and impact of human factors training for healthcare professionals or trainees who work in or interface with the OR environment. Titles, abstracts and full texts will be independently screened by two authors for eligible studies. Any disagreements will be resolved by discussion or by a third author when disagreement persists. Study information and training characteristics, such as the training tool used and type of learners and teachers, will be charted and summarised, and key themes in human factors training will be identified. Each training offering will be classified under the appropriate knowledge area(s) of human factors described by the Chartered Institute of Ergonomics \& Human Factors (CIEHF). Themes that are not captured by the CIEHF framework will be independently recorded by two authors and included based on group discussion and consensus.

Ethics and dissemination Research ethics board approval is not required for this scoping review. The findings of this study will be disseminated at local and national conferences and will be published in a peerreviewed journal.

\section{INTRODUCTION}

Healthcare is described as a complex and dynamic sociotechnical system, wherein the care delivered is dependent on the behaviours and actions of providers and the
Strengths and limitations of this study

- This will be the first scoping review to describe human factors for the operating room as applied by education or training offerings for clinical and nonclinical healthcare professionals.

- This review will be performed by evaluating multiple databases with a comprehensive search strategy to capture studies on human factors training for the operating room setting.

- Training content will be evaluated based on 67 different themes of human factors by author reviewers trained by a human factors expert and staff surgeon.

- Limitations of this study include a language restriction to the English language.

design of the system they work in. ${ }^{1}$ Adoption of human factors principles in healthcare has been suggested as a method to improve care delivery and patient safety. ${ }^{2}$ In general, the study of human factors encompasses interactions between individuals, the workplace and the tools and equipment used, while considering how they can be optimised to reduce and control errors. For example, safety checklists in surgery were developed from human factors principles to address the high rates of avoidable critical events and promote process improvement in the operating room (OR). ${ }^{3}{ }^{4}$ However, interventions based on human factors can fail without a complementary system or culture that supports these initiatives, especially when they are simply viewed as additional tasks to complete. ${ }^{5} 6$ Accordingly, more work is required to apply human factors principles in system architecture and organisational culture to advance patient safety, provider experience and efficiency. ${ }^{5-9}$

Human factors have been especially relevant in nuclear science and aviation industries where seemingly insignificant errors can 
lead to critical incidents. One recent example of such errors was the false missile alert in Hawaii that resulted from communication failures between employees, causing widespread panic, multiple emergency dispatch calls and vehicle accidents. ${ }^{10}$ In surgery, complex interactions between multiple personnel (eg, surgeons, anesthesiologists, nurses, other perioperative workers), different equipment and tools (eg, surgical devices, monitors) and OR administration (eg, access, staff availability) make it crucial yet challenging to effectively adopt and apply human factors principles to improve patient safety and provider experience. ${ }^{11}$ The critical nature of operative procedures adds vulnerability to errors that can constitute up to $79 \%$ of adverse events in healthcare. ${ }^{12}$ Moreover, the encapsulated setting of the OR, related to sterility and patient privacy, does not lend itself well to external examination. This amplifies the challenge around obtaining robust intraoperative analysis and participation in reflective quality improvement practices. ${ }^{13}$ As such, awareness around human factors has been shown to be heterogeneous among frontline surgical providers. ${ }^{6} 91415$

However, this heterogeneity cannot be attributed solely to individual or system resistance to human factors uptake. Intersecting between fields in engineering, psychology, medicine and other numerous disciplines, there is considerable variation and range in how human factors are applied in practice. ${ }^{916}$ Ergonomics, for example, is sometimes used interchangeably with human factors with a focus on workplace and product design, while other proponents consider human factors to capture a more psychological or cognitive sphere such as nontechnical skills. ${ }^{17-19}$ Likewise, various theoretical frameworks on the role of human factors in surgery have been described, ${ }^{20-22}$ but how they are being translated to actual practice in the OR and what is collectively being labelled under the concept of human factors in vivo remain unknown.

Effective and meaningful integration of human factors in surgical safety improvement efforts is highly dependent on education and knowledge translation of human factors for healthcare professionals. ${ }^{6}$ Currently, the International Ergonomics Association defines human factors as the scientific discipline concerned with the understanding of interactions among humans and other elements of a system. ${ }^{23}$ Analyses of flow disruptions in surgery have also demonstrated that factors beyond individual nontechnical skill errors, such as equipment and technology problems as well as issues in resource accessibility and poor systems organisation, can lead to patient safety incidents and a reduced provider experience. ${ }^{5}$ But whether human factors in the OR are being used to describe and address such concepts that span the individual, the workplace and the tools used, and whether healthcare professionals are being trained in these areas are unknown.

The objective of this scoping review is to describe human factors for the intraoperative environment as characterised by the content and tools used in human factors education or training for healthcare professionals and trainees who work within or interface with the OR.
The findings of this scoping review will demonstrate practical application of human factors and knowledge gaps among healthcare professionals in surgery. This will ultimately inform future human factors' integration, curriculum development and quality improvement to advance patient safety in the OR.

\section{METHODS}

A scoping review of the literature will be conducted to investigate the content, application and effect of individual interventions that are labelled as human factor training or education for the OR environment. This study will follow the Preferred Reporting Items for Systematic reviews and Meta-Analyses extension for Scoping Reviews guidelines. ${ }^{24}$ Development of this study will be informed by key stakeholders in human factors including a human factors expert (BT), clinicians from the OR (JAK, FB) and a health systems research expert (CK).

\section{Stage 1: identifying relevant training offerings}

The following electronic bibliographic databases have been searched in August 2020 in consultation with a research librarian who drafted and refined the search strategy with the research team: MEDLINE, Embase, PsycINFO, CINAHL, Health and Psychosocial Instruments and ERIC. These databases were searched from inception to August 2020, and no restrictions were applied. The search strategy combined the term 'human factors' with concepts related to 'training' (eg, teaching, education, intervention, course etc) and 'operating room' (eg, surgery, intraoperative, surgeon, nurse, anesthesiologist etc). The search strategy used for MEDLINE is included in the online supplemental appendix A. Reference lists of included studies will be hand checked to ensure that all relevant literatures have been identified. In addition, a research librarian will provide a list of key articles in human factors in surgery that will be hand checked and screened for inclusion or exclusion.

\section{Stage 2: study selection}

Titles and abstracts of studies retrieved by the search strategy will be independently screened by two authors who will identify articles that potentially meet the inclusion criteria. Full-text articles of the potentially eligible studies will then be retrieved and independently assessed for eligibility. Any disagreements will be resolved by discussion or input from a third author if disagreement persists. Reasons for excluding studies will be documented. A preliminary search of PROSPERO, MEDLINE and Embase was previously conducted and no systematic or scoping reviews on the topic were identified. The eligibility criteria are described in terms of the population, concept and context as recommended by the guidelines. 
Table 1 CIEHF knowledge areas of human factors and ergonomics

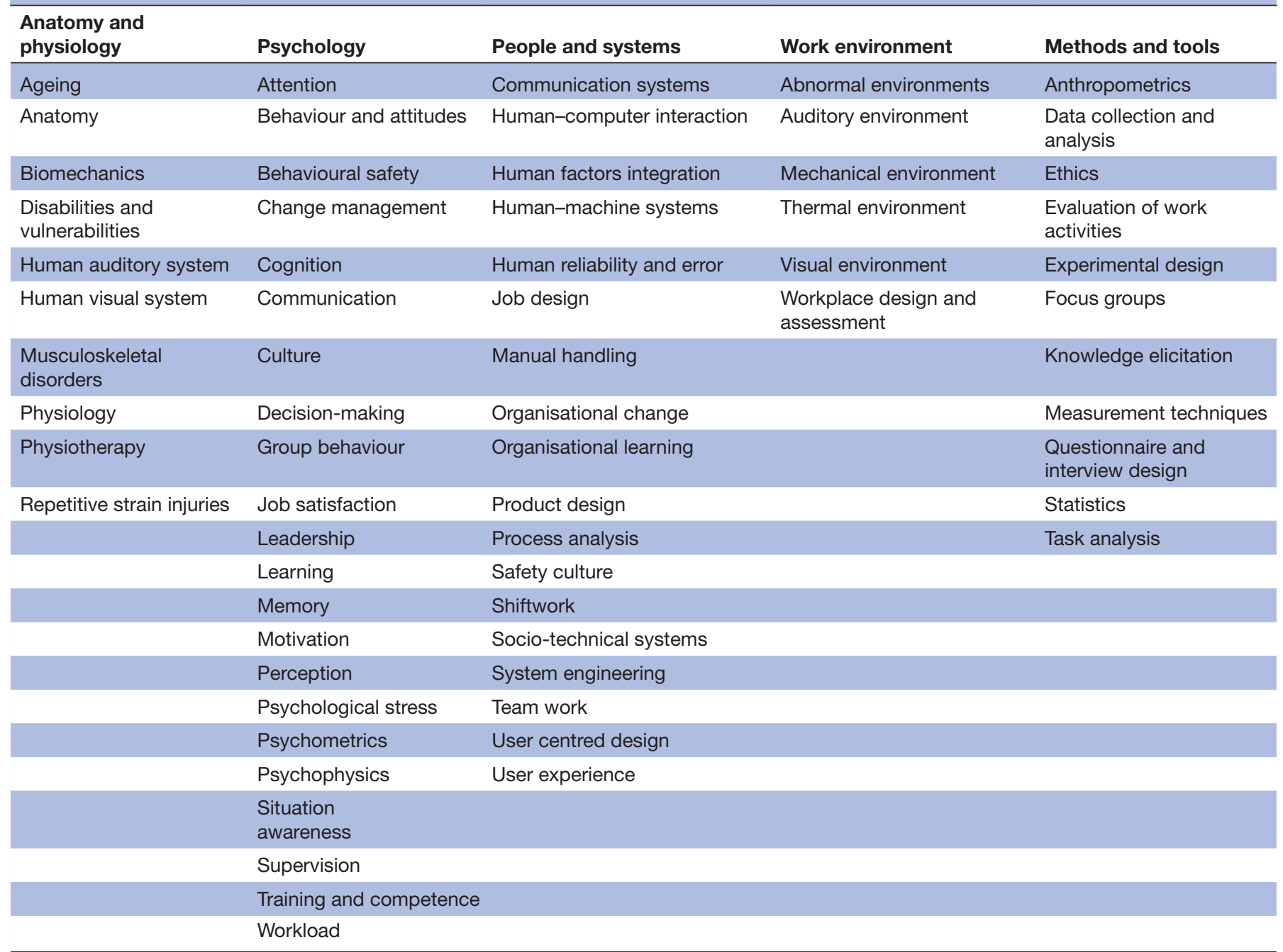

CIEHF, Chartered Institute of Ergonomics \& Human Factors.

\section{Inclusion criteria}

- Population: clinical (eg, surgeons, nurses etc) and nonclinical (eg, OR administrators, housekeeping staff, hospital porter etc) personnel who work in and/ or directly interface with the OR.

- Concept: any individual education or training intervention that includes teaching labelled under 'human factors' for the OR setting.

- Context: original research articles published in any year in the English language including single-arm studies, double-arm studies, qualitative and quantitative studies, randomised controlled trials and quasiexperimental studies.

\section{Exclusion criteria}

- Studies that do not report original data (eg, editorials, commentaries) and conference abstracts.

- Studies that only report theoretical considerations or reviews of human factors or human factors training (references will be hand checked).
- Studies that do not report the content of the human factors training offering.

- Studies without a component specific to the OR environment (eg, surgical ward, emergency department etc).

\section{Stage 3: data charting}

Two authors will independently chart data from the included articles onto a standardised data spreadsheet using Microsoft Excel V.16.46, which will be piloted by the authors with example papers to provide necessary calibration and ensure accuracy. Data will be charted by two authors and any disagreements will be resolved through discussion or with input from a third author when disagreement persists. Charted information will include:

- Study information: author(s) information, year of publication, country of origin, article keywords, study type.

- Training participants: number, type of and level of training of learner(s) (eg, surgeons, nurses, residents etc), number and type of teacher(s) (eg, human 
factors experts etc), interdisciplinary versus intradisciplinary learning.

- Training design: type of training developers (eg, psychologists, airline pilots etc), type of training mediums or tools used (eg, presentations, simulations, briefing etc), length of training, frequency of training, assessment tool and type used (eg formative, summative, behaviour rating systems etc).

- Training content: learning objectives, skills or concepts taught (eg, communication, situational awareness, ergonomics etc), quantitative or qualitative outcomes measured or described, feedback from participants.

- Training cost.

\section{Stage 4: summarising and reporting results}

Collected data will be presented in a tabular or diagrammatic form along with a narrative summary in a manner that aligns with the objectives and scope of this review. Study characteristics including author information, year of publication, location of the research institution and study type will be summarised. Training participants and design, including learner type and number, trainer type and number, training duration and frequency, training format, training assessment and training outcome will also be summarised.

A narrative synthesis of training content will be provided based on the learning objectives and skills or concepts taught in each training offering. Training content will be further summarised by evaluating each study according to the 67 knowledge areas of human factors and ergonomics as described by the Chartered Institute of Ergonomics \& Human Factors (CIEHF) (table 1). ${ }^{20}$ These knowledge areas represent different components or categories of human factors as defined by CIEHF, some of which may or may not apply to the current adoption and use of the term human factors for the OR setting. For the purpose of this study, the CIEHF framework will be used to characterise human factors for the OR based on the most frequently observed knowledge areas among the included studies. Two authors will be trained by a human factors expert and staff surgeon on the CIEHF framework to classify each training offering under the appropriate knowledge area(s). Inter-rater reliability will be assessed using Cohen's kappa statistics. A calibration session will be held before to ensure accuracy and reproducibility in classifying knowledge areas. The two authors will also independently record any themes that may not have been captured by the CIEHF framework. These themes along with any disagreements between the two authors when categorising the included studies will be resolved through group discussion and consensus. A quantitative meta-analysis and a formal assessment of methodological quality will not be performed due to the anticipated heterogeneity among the included studies and the review's focus on defining the current scope of HF training for the operative setting.

\section{Patient and public involvement}

No patient or public involvement was included in this study.

\section{ETHICS AND DISSEMINATION}

This study does not require research ethics board approval. The study will be completed in June 2021. Findings will be disseminated through presentations at academic conferences and will also be submitted for publication in a peer-reviewed journal. Finally, this scoping review will help identify opportunities and knowledge gaps that can be the focus of future research activity and medical curriculum development.

\section{Author affiliations}

${ }^{1}$ University of Ottawa Faculty of Medicine, Ottawa, Ontario, Canada

${ }^{2}$ MedLed Ltd, Slough, UK

${ }^{3}$ Department of Epidemiology, Biostatistics and Occupational Health, McGill University Faculty of Medicine, Montreal, Quebec, Canada

${ }^{4}$ Saegis, Canadian Medical Protective Association, Ottawa, Ontario, Canada

${ }^{5}$ Division of General Surgery, Department of Surgery, University of Ottawa Faculty of Medicine, Ottawa, Ontario, Canada

${ }^{6}$ Office of Research Services and School of Business, MacEwan University School of Business, Edmonton, Alberta, Canada

Contributors $A L$ and $F B$ conceived the study. $A L$ and $F B$ developed the research question and drafted the protocol. AF, AR, BT, CK, FB, JAK and KD provided advice and edits on the final protocol submission. All authors proof-read and approved the final protocol manuscript.

Funding This protocol was supported by funding from the NSERC Discovery Grant (RGPIN-2019-04884).

Competing interests FB is the Director at Saegis, a subsidiary of the Canadian Medical Protective Association. KD is the Programme Lead at Saegis. BT is the Founder and Managing Director at MedLed Ltd.

Patient consent for publication Not required.

Provenance and peer review Not commissioned; externally peer reviewed.

Supplemental material This content has been supplied by the author(s). It has not been vetted by BMJ Publishing Group Limited (BMJ) and may not have been peer-reviewed. Any opinions or recommendations discussed are solely those of the author(s) and are not endorsed by BMJ. BMJ disclaims all liability and responsibility arising from any reliance placed on the content. Where the content includes any translated material, BMJ does not warrant the accuracy and reliability of the translations (including but not limited to local regulations, clinical guidelines, terminology, drug names and drug dosages), and is not responsible for any error and/or omissions arising from translation and adaptation or otherwise.

Open access This is an open access article distributed in accordance with the Creative Commons Attribution Non Commercial (CC BY-NC 4.0) license, which permits others to distribute, remix, adapt, build upon this work non-commercially, and license their derivative works on different terms, provided the original work is properly cited, appropriate credit is given, any changes made indicated, and the use is non-commercial. See: http://creativecommons.org/licenses/by-nc/4.0/.

ORCID iD

Alex Lee http://orcid.org/0000-0003-3842-1225

\section{REFERENCES}

1 Carayon P, Bass E, Bellandi T, et al. Socio-Technical systems analysis in health care: a research agenda. IIE Trans Healthc Syst Eng 2011;1:145-60.

2 et alFlin R, Winter J, Sarac C. Human factors in patient safety: review of topics and tools. World Health Organization, 2009. Available: https://www.who.int/patientsafety/research/methods measures/ human_factors/human_factors_review.pdf [Accessed 24 Apr 2020]. 
3 Carayon P, Xie A, Kianfar S. Human factors and ergonomics as a patient safety practice. BMJ Qual Saf 2014;23:196-205.

4 O'Connor P, Reddin C, O'Sullivan M, et al. Surgical checklists: the human factor. Patient Saf Surg 2013;7:14.

5 Shouhed D, Gewertz B, Wiegmann D, et al. Integrating human factors research and surgery: a review. Arch Surg 2012;147:1141-6.

6 Gurses AP, Ozok AA, Pronovost PJ. Time to accelerate integration of human factors and ergonomics in patient safety. BMJ Qual Saf 2012;21:347-51.

7 National Academies of Sciences, Engineering and M. Factors Contributing to Clinician Burnout and Professional Well-Being. In: Taking action against clinician burnout: a systems approach to professional well-being. The National Academies Press, 2019.

8 Ghaferi AA, Myers CG, Sutcliffe KM. The next wave of hospital innovation to make patients safer. Harv Bus Rev 2016;94.

9 Russ AL, Fairbanks RJ, Karsh B-T, et al. The science of human factors: separating fact from fiction. BMJ Qual Saf 2013;22:802-8.

10 Russo CH. Heart Attack, Car Crash: Hawaii's Missile Alert Triggered Some Damage. Huffington Post.

11 Palmer G, Abernathy JH, Swinton G, et al. Realizing improved patient care through human-centered operating room design: a human factors methodology for observing flow disruptions in the cardiothoracic operating room. Anesthesiology 2013;119:1066-77.

12 Schwendimann R, Blatter C, Dhaini S, et al. The occurrence, types, consequences and preventability of in-hospital adverse events - a scoping review. BMC Health Serv Res 2018;18:521.

13 Jung JJ, Jüni P, Lebovic G, et al. First-Year analysis of the operating room black box study. Ann Surg 2020;271:122-7.
14 Fann JI, Moffatt-Bruce SD, DiMaio JM, et al. Human factors and human nature in cardiothoracic surgery. Ann Thorac Surg 2016;101:2059-66.

15 Hilt AD, Kaptein AA, Schalij MJ, et al. Teamwork and safety attitudes in complex aortic surgery at a Dutch Hospital: cross-sectional survey study. JMIR Hum Factors 2020;7:e17131.

16 Cacciabue PC. Guide to applying human factors methods. 1st ed. London: Springer-Verlag, 2004.

17 What is ergonomics? Chartered Institute of Ergonomics \& Human Factors.

18 ISO/TC 159. Ergonomics principles in the design of work systems, 2016.

19 Introduction to human factors. health and safety executive.. Available: https://www.hse.gov.uk/humanfactors/introduction.htm [Accessed $01 \mathrm{Jul} 2020]$.

20 D'Addessi A, Bongiovanni L, Volpe A, et al. Human factors in surgery: from three mile island to the operating room. Urol Int 2009;83:249-57.

21 EIBardissi AW, Sundt TM. Human factors and operating room safety. Surg Clin North Am 2012;92:21-35.

22 Casali G, Cullen W, Lock G. The rise of human factors: optimising performance of individuals and teams to improve patients outcomes. J Thorac Dis 2019;11:S998-1008.

23 International Ergonomics Association. Definition, domains of specialization, systemic approach. Available: https://iea.cc/definitionand-domains-of-ergonomics/ [Accessed 06 Mar 2021].

24 Tricco AC, Lillie E, Zarin W, et al. PRISMA extension for scoping reviews (PRISMA-ScR): checklist and explanation. Ann Intern Med 2018;169:467-73. 